\title{
Human sperm handling in intracytoplasmic sperm injection processes: In vitro studies on mouse oocyte activation, embryo development competence and sperm oxidation-reduction potential
}

\author{
S. Roychoudhury, I. Maldonado-Rosas, A. Agarwal, S. C. Esteves, R. Henkel and R. Sharma
}

\section{Summary}

Polyvinylpyrrolidone (PVP) and hyaluronic acid (HA) are routinely used in handling spermatozoa for intracytoplasmic sperm injection (ICSI). As there are still concerns about possible adverse effects on the embryo, this study investigated sperm handling in a mouse ICSI model to (i) evaluate oocyte activation after injection of spermatozoa selected for rotational or linear motion in PVP; (ii) assess the effect of sperm selection in PVP, HA and medium on oocyte activation; (iii) examine the effects of PVP and HA on parthenogenetic oocyte activation and embryo development; and (iv) assess the oxidation-reduction potential (ORP) of spermatozoa exposed to PVP, HA or medium. Oocyte activation was higher when spermatozoa exhibited rotational motion rather than linear motion ( $79 \%$ vs. $52 \% ; p=.05$ ). There was no difference in oocyte activation and embryo development after parthenogenetic oocyte activation after sperm injection using PVP, HA or medium-incubated spermatozoa. PVP-selected spermatozoa exhibited lower $(p<$. .ooo1) ORP levels than using HA. Thus, results indicate that the sperm handling method and the type of medium used impact ICSI outcomes. Overall, sperm incubation in PVP, HA and medium yields similar outcomes with regard to oocyte activation and embryo development. However, PVP provides more antioxidative protection than HA and should therefore be preferred for sperm manipulation.

\section{Introduction}

Intracytoplasmic sperm injection (ICSI) is used to overcome severe male factor infertility, failed in vitro fertilisation (IVF) and unexplained infertility, among other conditions (Bungum, Bungum, Humaidan, \& Andersen, 2004; Devroey \& Van Steirteghem, 2004; Takeuchi et al., 2000). Fertilisation rates after sperm injection vary from 50\% to 80\% (Neri, Lee, Rosenwaks, Machaca, \& Palermo, 2014), and these rates are dependent not only on oocyte quality but also on sperm quality and sperm source (Majumdar \& Majumdar, 2013; Montag, Toth, \& Strowitzki, 2012). As a matter of fact, insertion of the microinjection pipette into the oocyte cytoplasm by itself is not responsible for oocyte activation (Tesarik, Sousa, \& Testart, 1994).

For fertilisation to take place, spermatozoa must be able to activate the oocyte. Therefore, an integral laboratory aspect of ICSI is sperm selection, which is usually based on viability-often expressed by progressive or in situ motility and normal morphology (Montag et al., 2012). In 
mouse oocytes, Kimura et al. (1998) reported that sperm-borne oocyte-activating factors (SOAF) appear during transformation of the round spermatid into the spermatozoon. However, the action of SOAF is not species-specific as mouse oocytes can be activated by injecting spermatozoa from foreign species, including hamster, rabbit, pig, human and fish. This is advantageous because unlike human oocytes, mouse oocytes are widely available and can be used in an experimental ICSI model.

During the journey through the female reproductive tract and before reaching the oocyte, spermatozoa exhibit a variety of motility modes and swimming patterns, including linear as well as rotational motion or beat frequency (Hz) (Nosrati, Driouchi, Yip, \& Sinton, 2015; Subramani, Basu, Thangaraju, \& Dandekar, 2014). In order to facilitate the selection, handling and immobilisation of the male gamete prior to ICSI, post-washed sperm specimens are usually loaded into a microdroplet containing a viscous medium, such as polyvinylpyrroli-done (PVP) or hyaluronic acid (HA) that slowdown sperm movement. Increased medium viscosity dampens the strong linear component (Nosrati et al., 2015) and may convert sperm movement into rotational motion that is believed to be the result of a reversible change in the molecular components of the mitochondrial apparatus (Subramani et al., 2014). Nevertheless, it is at present unclear whether spermatozoa selected for ICSI displaying progressive and rotational motion into a PVP drop have better oocyte activation potential than those with progressive and nonrotational (linear) motion. This is an interesting concept that deserves further investigation because sperm rotational motion is easy to observe into the high viscosity media such as PVP using a regular inverted microscope (40x).

Notwithstanding, concerns about the safety of PVP (Bourne et al., 1995) and its possible adverse impact on embryo development have also been raised (Kato \& Nagao, 2012). It has been reported that spermatozoa selected in PVP exhibit a delay in calcium oscillations after oocyte injection, which may prevent nuclear decondensation (Clarke \& Johnson, 1988; Dozortsev, Rybouchkin, De Sutter, Qian, \& Dhont, 1995). Additionally, impairment in the development of two-cell mouse embryos (Bergers et al., 1993) and direct toxicity of PVP when injected into mouse zygotes (Bras, 1994) was noted. On the contrary, PVP was noted to have no mutagenic potential as analysed by the frequency of sister chromatid exchange (Ray, Howell, Mcdermott, \& Hull, 1995). Additionally, PVP was found harmless to the quality and development of bovine embryos (Motoishi, Goto, Tomita, Ookutsu, \&Nakanishi,1996).

As HA-based products are commercially available, a few reports indicated that such medium might represent a good physiologic alternative to PVP (Balaban et al., 2003; Barak, Menezo, Veiga, \& Elder, 2001; Parmegiani, Cognigni, Bernardi, et al., 2010; Parmegiani, Cognigni, Ciampaglia, et al., 2010). In vivo human oocytes are surrounded by HA and only the mature spermatozoa that have extruded specific HA receptors are able to reach and fertilise the oocyte (Parmegiani, Cognigni, Ciampaglia, et al., 2010). In vitro spermatozoa bound to HA are believed to be those that have completed plasma membrane remodelling, cytoplasmic extrusion, and nuclear maturation (Cayli et al., 2003; Huszar et al., 2007). However, the literature is mixed with regard to the advantage of using HA instead of PVP, therefore indicating the need for further studies (Balaban et al., 2003; Barak et al., 2001; Choe etal., 2012; Majumdar\&Majumdar, 2013). 
After sperm injections, total fertilisation failure occurs in about $1 \%-3 \%$ cycles and the phenomenon can repeat in subsequent cycles (Flaherty, Payne, \& Matthews, 1998; Kashir et al., 2010). It has been hypothesised that total fertilisation failure might be due to a sperm-related oocyte activation deficiency (Flaherty et al., 1998; Neri et al., 2014; Swain \& Pool, 2008; Yanagida, 2004). In this regard, mouse oocytes provide a useful model for the assessment of sperm-associated oocyte activation factor (Rybouchkin, Dozortsev, Pelinck, De Sutter, \& Dhont, 1996) because they are tolerant to $\mathrm{Ca}^{2+}$ oscillation patterns (Ozil et al., 2005; Toth, Huneau, Banrezes, \& Ozil, 2006). Parthenogenetic mouse oocyte activation relies on the calcium wave that generates the responses associated with fertilisation when calcium release reaches a certain threshold (Uranga, Pedersen, \& Arechaga, 1996). Calcimycin also known as A23187 is a $\mathrm{Ca}^{2+}$-selective ionophore widely used as an oocyte activation agent (Kashir et al., 2010). It can induce $\mathrm{Ca}^{2+}$ influx by altering the plasma membrane permeability or acting directly on intracellular organelles that release $\mathrm{Ca}^{2+}$ (Mason \& Grinstein, 1993; Morgan \& Jacob, 1994).

Lastly, oxidative stress has been recognised as an important contributory factor to male infertility (Agarwal, Ahmad, \& Sharma, 2015; Agarwal et al., 2014; Roychoudhury, Sharma, Sikka, \& Agarwal, 2016; Tremellen, 2008). In ICSI, seminal plasma is removed during semen processing, but toxic oxygen metabolites (generated by immature spermatozoa and leucocytes) might still attack the sperm devoid of protective seminal plasma antioxidants (Aitken \& Baker, 1995; Zini, San Gabriel, \& Baazeem, 2009). A new technique called MiOXSYS system allows the assessment of oxidationreduction potential (ORP). It provides a comprehensive measure of seminal oxidative stress and is indicative of the overall balance between all available oxidants and antioxidants in a given sample (Agarwal, Roychoudhury, Bjugstad, \& Cho, 2016; Agarwal, Sharma, Roychoudhury, Du Plessis, \& Sabanegh, 2016). To our knowledge, the ORP of spermatozoa selected for ICSI after exposure to PVP and HA has not been measured yet.

To investigate these several events that take place prior to sperm injection, we conducted a series of experiments using both an ICSI experimental model and a new method for sperm selection. Our objectives were to assess (i) the activation of mouse oocytes after ICSI using human spermatozoa showing progressive motility with linear or rotational motion independent of the type of rotation (clockwise or anticlockwise); (ii) the activation of mouse oocytes after ICSI of human spermatozoa selected in PVP and HA microdroplets; (iii) the activation and embryo developmental competence of the parthenogenetically activated mouse oocytes after ICSI using PVP and HA; and lastly (iv) the ORP of human spermatozoa selected for ICSI after exposure to PVP and HA. These experiments will help answer if there is any advantage of using spermatozoa exhibiting rotational movement and answer the debate on the value of using PVP vis-a-vis the need to replace with HA for optimal selection of spermatozoa prior to ICSI. The measurement of ORP in prepared spermatozoa selected for ICSI following exposure to PVP or HA will further help identify the media with low oxidative stress. 


\section{2 | Materials and Methods}

Following Institutional approval, discarded semen samples were used. All subjects provided a written informed consent. Frozen mouse oocytes were procured from a vendor (Embryotech, Haverhill, MA), and hence, no ethical clearance was necessary from the Institutional Animal Care and Use Committee.

\section{1 | Experiment 1. Activation of mouse oocytes after intracytoplasmic injection of human spermatozoa with linear and rotational motion}

Fresh human semen samples $(n=2)$ from fertile normozoospermic men according to 2010 WHO guidelines and $\mathrm{B}_{6} \mathrm{C}_{3} \mathrm{~F}_{1}$ frozen metaphase II mouse oocytes ( $n=88$; Embryotech, Haverhill, MA) were used in this experiment. Spermatozoa were prepared by the density gradient centrifugation with $40 \%$ and $80 \%$ gradients (Cooper Surgical, Trumbull, CT, USA) and selected for ICSI based on either linear or rotational motion in deep regions of a PVP (Origio, Denmark) microdroplet. Spermatozoa with linear motion were those that changed their position but not their location by exhibiting a clear two-dimensional movement in the bottom of a PVP droplet under inverted microscope (Leica Microsystems, Wetzlar, Germany) examination using a $40 \times$ objective. On the other hand, spermatozoa with rotational motion were the ones that changed position by gyrating over its own axis under the same aforementioned conditions (Figure 1). Frozen mouse oocytes were thawed, and the surviving oocytes $(n=81)$ were randomly assigned into two groups for microinjection. Spermatozoa with linear motion (Group 1; $n=39$ ) and rotational motion (Group 2; $n=42$ ) were selected and microinjected into the oocyte cytoplasm. In both cases, spermatozoa displaying progressive motility and rotational or linear motion were selected under $40 \times$ objective. Using the same magnification, selected spermatozoa were immobilised by squeezing the tail using the injection micropipette prior to injection. After sperm injections, the oocyte activation rates (recorded as a percentage of embryo development at the two-cell stage $24 \mathrm{hr}$ after microinjection) were assessed (Figure 2). Culture was performed under standard culture conditions.

\section{2 | Experiment 2. Activation of mouse oocytes after intracytoplasmic injection of human spermatozoa selectedin PVPandHAdroplets}

Fresh human semen samples $(n=2)$ from fertile normozoospermic men according to 2010 WHO guidelines and $\mathrm{B}_{6} \mathrm{C}_{3} \mathrm{~F}_{1}$ frozen metaphase II mouse oocytes $(n=94)$ were used in this experiment. Spermatozoa prepared by the density gradient centrifugation were selected for ICSI in either a microdroplet of PVP (Origio, Denmark) or HA (SpermCatch, Nidacon, Sweden) or culture medium (Quinn's Advantage medium with HEPES, Origio, Denmark; control). Similar to PVP, the HA-containing product slows sperm motility sufficiently for the spermatozoa to be captured with an injection pipette (Balaban et al., 2003). Surviving oocytes $(n=83)$ were randomised for ICSI according to the medium used for sperm selection: PVP $(n=28)$; HA $(n=27)$ and culture medium (control; $n=28$ ). Sperm immobilisation and injection were carried out as described in experiment 1. After ICSI, the oocyte activation rates (recorded as a 
percentage of surviving oocytes at the two-cell stage $24 \mathrm{hr}$ after microinjection) were reported (Figure 3).

\section{3 | Experiment 3. Parthenogenetic activation and development competence of mouseoocytes after intracytoplasmic injection of PVP or HA}

$\mathrm{B}_{6} \mathrm{C}_{3} \mathrm{~F}_{1}$ frozen metaphase II mouse oocytes $(n=116)$ were used in this experiment. Frozen mouse oocytes were thawed, and the surviving oocytes $(n=102)$ were randomised into three groups for microinjection: PVP-treated $(n=36)$; HA-treated $(n=34)$ and control $(n=32)$. In the respective groups, 2-3 picolitres of PVP, HA or culture medium was microinjected into the oocyte cytoplasm (Motoishi et al., 1996). Of note, no spermatozoa were injected. Immediately after microinjection, the oocytes were parthenogenetically activated by exposure to a ready-to-use calcimycin $\left(\mathrm{Ca}^{2+}\right.$-selective ionophore $)$ A23187 solution (GM508 Cult-Active, Gynemed, Germany) for 15 min. The parthenogenetic activation of mouse oocytes and embryo development were recorded until the blastocyst stage (Figure 4).

FIGURE 1 Linear and rotational motion of human spermatozoa. (a) Spermatozoa with linear motion change their position but not location by exhibiting twodimensional movement in the bottom of a polyvinylpyrrolidone microdroplet, and (b) spermatozoa with rotational motion that change their position by gyrating over its own axis

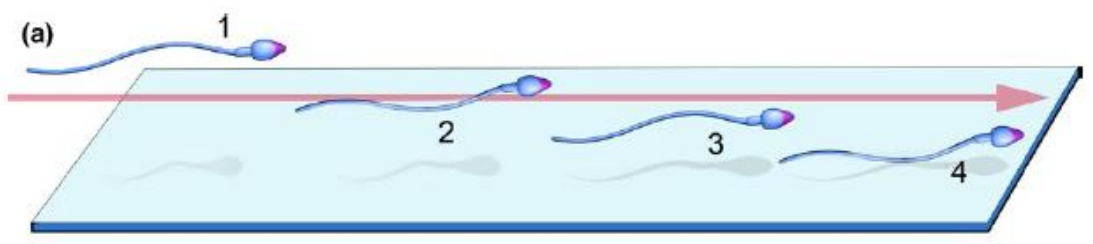

(b)

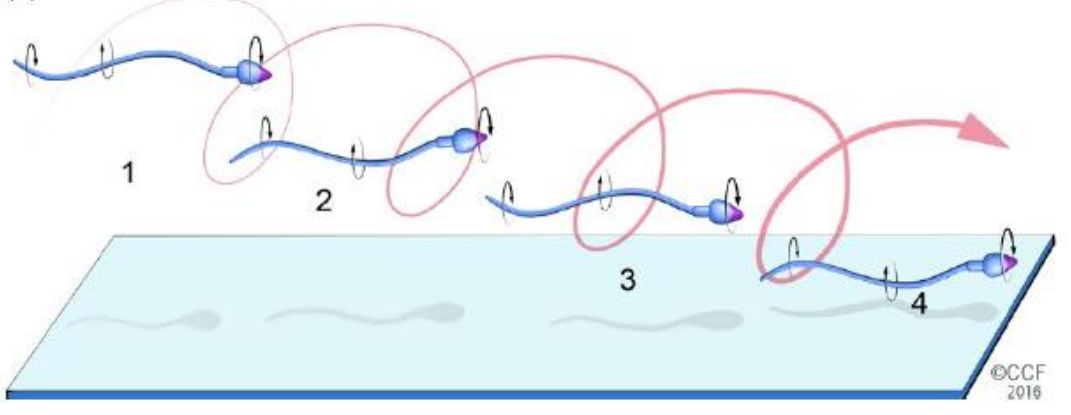




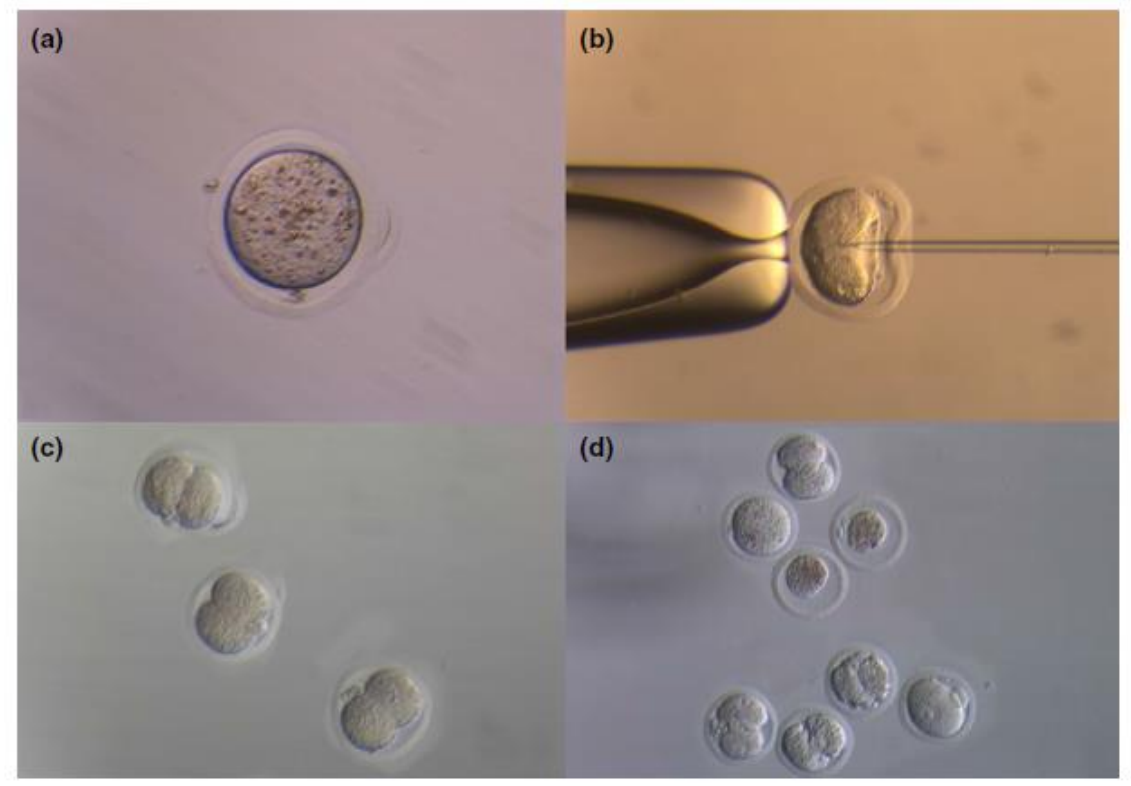

FIGURE 2 Activation of mouse oocytes after intracytoplasmic sperm injection of human spermatozoa with linear or rotational motion: (a) metaphase II stage mouse oocyte, (b) Intracytoplasmic injection, (c) embryo development at the two-cell stage, (d) surviving embryos at the two-cell stage

\section{4 | Experiment 4. Oxidation-reduction potential of human spermatozoa selected for ICSI after PVP or HA exposure}

Fresh human semen samples $(n=11)$ from normozoospermic men according to 2010 WHO guidelines were used in this experiment. Spermatozoa were prepared by density gradient centrifugation, and the washed sperm preparations were adjusted to $5 \times 10^{6}$ sperm $/ \mathrm{ml}$. A sperm aliquot was then loaded into a microdroplet of either PVP orHA or sperm wash medium in a 3:10 ratio (vol./vol.). The sORP (static ORP which refers to the passive or current state of activity between oxidants and antioxidants) was measured after 20 min and 1 $\mathrm{hr}$ of exposure using a galvanostat-based technology-the MiOXSYS System (Aytu Bioscience, Englewood, CO) in triplicate at room temperature (Figure 5). The MiOXSYS system was calibrated prior to testing according to the manufacturer's instructions. For analysing the sample, $30 \mu \mathrm{l}$ of sperm suspension was applied to the sample application port on the inserted sensor. After 3-4 min, audible beeps indicated the completion of the test and the absolute value of sORP was displayed in millivolts or $\mathrm{mV}$ on the screen (Agarwal, Sharma, et al., 2016). The sORP value was then divided by sperm concentration to obtain normalised sORP, which was expressed as $\mathrm{mV} / 10^{6} \mathrm{sperm} / \mathrm{ml}$.

For all experiments, one well-trained scientist (SR) performed all sperm handling procedures whereas another proficient scientist (IMR) performed all injections.

\section{5 | Statistical analysis}

For experiments 1-3, chi-square or Fisher exact tests as appropriate were used to compare the outcome measures (expressed as mean $\pm 95 \%$ confidence interval) among the groups using an alpha level of $p<.05$. In experiment 4 , the ORP data of human spermatozoa selected for ICSI after exposure to PVP, HA or culture medium were normalised as $\mathrm{mV} / 10^{6} \mathrm{sperm} / \mathrm{ml}$ and expressed as mean $\pm S D$. In the latter, the paired $t$ test was used to compare the groups and differences were considered statistically significant at a level $p<.05$. 


\section{3. $\quad$ Results}

3.1 Experiment 1. Activation of mouse oocytes after intracytoplasmic injection of human spermatozoa with progressive motility and either linear or rotational motion

The oocyte activation rates following sperm selection by linear versus rotational motion are presented in Figure 6. The oocyte activation rate achieved in the group of rotational motion was $79.2 \%$ compared to $52.3 \%(p=.05)$.

\section{2 | Experiment 2. Activation of mouse oocytes after intracytoplasmic injection of human spermatozoa selected in PVP or HA}

The rates of oocyte activation after intracytoplasmic injection of human spermatozoa selected in PVP, HA or culture medium (control) are presented in Figure 7. The oocyte activation rates were 73.7\% (14/19) in PVP group, 72.2\% (13/18) in HA group and 75.0\% (15/20) in the control group. These parameters were not significantly different among the groups.

FIGURE 3 Activation of mouse oocytes after intracytoplasmic sperm injection of human spermatozoa selected in droplet of polyvinylpyrrolidone, hyaluronic acid or culture medium (control): (a) metaphase II stage mouse oocyte, (b) intracytoplasmic injection, (c and d) surviving embryo(s) at the two-cell stage, ((e-f)) surviving twocell stage embryo and oocytes without any cleavage, $(\mathrm{g})$ surviving two-cell stage embryos at the two-cell stage

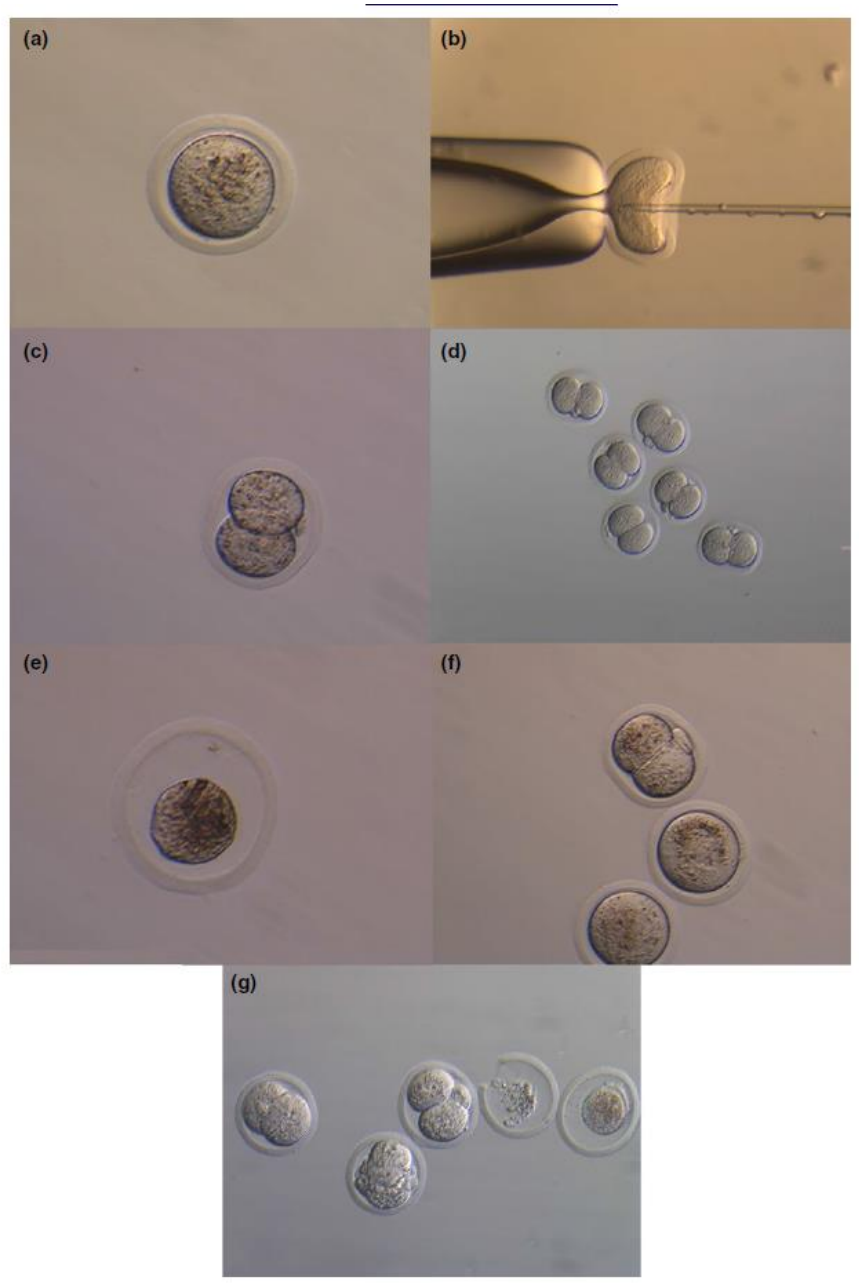




\section{3 | Experiment 3. Parthenogenetic activation and development competence of mouseoocytes after intracytoplasmicinjection with PVP and HA}

The development competence of parthenogenetically activated mouse oocytes after intracytoplasmic injection of PVP, HA or culture medium is presented in Figure 8. The oocyte activation rates were not significantly different among the groups: $62.5 \%(15 / 24)$ in the PVP group, 68.0\% (17/25) in the HA group and 61.9\% (13/21) in the control group. Of the embryos that reached the two-cell stage in the PVP, HA and control groups respectively, 53.3\% (8/15), 52.9\% (9/17) and 53.8\% (7/13) attained a four-cell stage. And among the embryos that reached the fourcell stage in the respective aforementioned groups, 53.3\% (8/15), 52.9\% (9/17) and 46.1\% (6/13) achieved the compacted morula stage. Lastly, on day 5 the blastocyst formation rates were $33.3 \%$ (5/15), 35.3\% (6/17) and 38.5\% (5/13) in the PVP, HA and control groups respectively. No significant differences were seen among the groups with regard to these outcome measures.

\section{4 | Experiment 4. Oxidation-reduction potential of human spermatozoa selected for ICSI after PVP and HA exposure}

The ORP of human spermatozoa selected for ICSI after exposure to PVP, HA or culture medium (control) is presented in Figure 9. The sORP levels differed significantly among the three groups both after $20 \mathrm{~min}$ and $1 \mathrm{hr}$ of exposure $(p<.0001)$. The lowest sORP levels were noted in the PVP-treated group followed by the HA-treated group and control group at both time points. Prior to any treatment with PVP or HA (Time o), sORP level in the control group was $57.60 \pm 0.63 \mathrm{mV} / 10^{6} \mathrm{sperm} / \mathrm{ml}$. After 20 min of incubation in PVP, HA and culture media (control), sORP levels were $46.27 \pm 1.96 \mathrm{mV} / 10^{6}$ sperm $/ \mathrm{ml}(p<.0001), 50.88 \pm 1.38 \mathrm{mV} / 10^{6} \mathrm{sperm} / \mathrm{ml}(p<.0001)$ and $57.49 \pm 2.55 \mathrm{mV} / 10^{6}$ sperm $/ \mathrm{ml}(p=.8909)$ respectively. These values did not change significantly after $1 \mathrm{hr}$ of exposure and were $47.04 \pm 1.01 \mathrm{mV} / 10^{6} \mathrm{sperm} / \mathrm{ml}(p<.0001), 50.80 \pm 1.23 \mathrm{mV} / 10^{6}$ sperm $/ \mathrm{ml}(p<.0001)$ and 57.30 $\pm 2.66 \mathrm{mV} / 10^{6} \mathrm{sperm} / \mathrm{ml}(p=.7197)$ in PVP, HA and control groups respectively. However, compared to the readings after 20 min, sORP values did not change in PVP $(p=.1020)$, HA $(p=.6918)$ and the control groups ( $p=.4761)$ after $1 \mathrm{hr}$. 


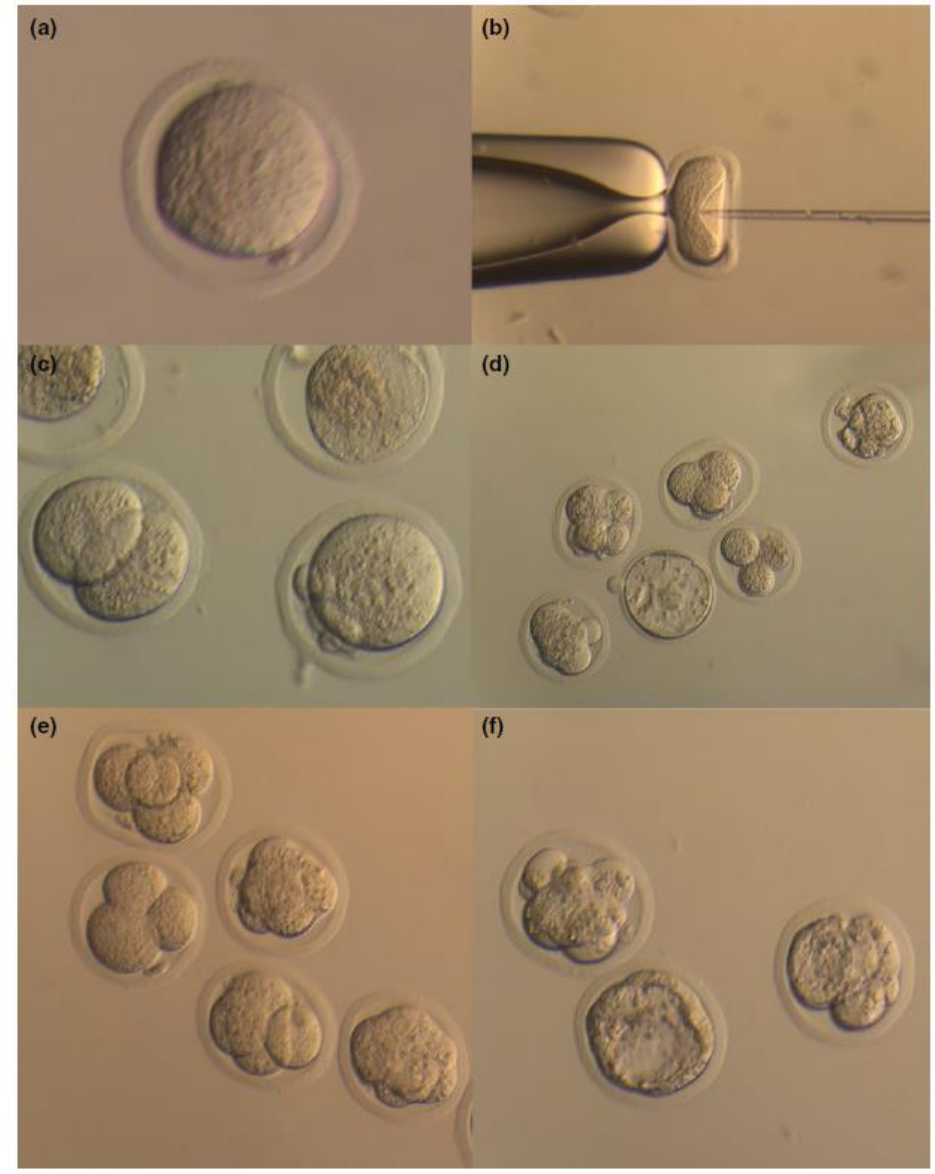

FIGURE 4 Development competence of parthenogenetically activated mouse oocytes after intracytoplasmic sperm injection (ICSI) of polyvinylpyrrolidone, hyaluronic acid or culture medium (control): (a) metaphase II stage mouse oocyte, (b) ICSI, (c) surviving embryo at the two-cell stage, (d) embryo development at the two-cell, three-cell, compacted morula and blastocyst stages, (e) embryo development at the two-cell and compacted morula stages, (f) embryo development at the compacted morula and blastocyst stages

\section{4 | Discussion}

Increased oocyte activation rates were achieved when mouse oocytes were injected with human spermatozoa exhibiting progressive motility and rotational motion over those with linear motion, suggesting that the mode of sperm movement at the time of selection might enhance sperm selection and influence ICSI outcome. Miki and Clapham (2013) proposed that rheotaxis, that is a taxis whereby an organism is turning into an oncoming current, is a major determinant of sperm guidance over long distances in the female reproductive tract. Moreover, sperm rheotaxis requires rotational motion. The tendency of human spermatozoa to exhibit rotational movement increases with the viscosity of the media, which dampens the strong transverse component and enables spermatozoa to more closely approach to, and align with the surface (Nosrati et al., 2015). Moreover, in ICSI where sperm quality is one of the decisive factors to oocyte fertilisation, embryo development, and implantation, distinguishing spermatozoa on the basis of beat frequency above $5.6 \mathrm{~Hz}$ has been suggested as an important tool in sperm sorting and selection (Subramani et al., 2014). To our knowledge, our study is the first to examine under a regular inverted microscope and into PVP droplet prior to injection the effect of sperm selection based on the linear or rotational motion characteristics. The biological plausibility of our findings is in accordance to the type of sperm movement found in the highly viscous fluid of confined regions of the fallopian tube (Nosrati et al., 2015). 
(a)

FIGURE 5 Measurement of oxidationreduction potential. (a) MiOXSYS Analyzer (placement of the sensor strip); (b) insertion of the sensor strip; (c) sensor in place

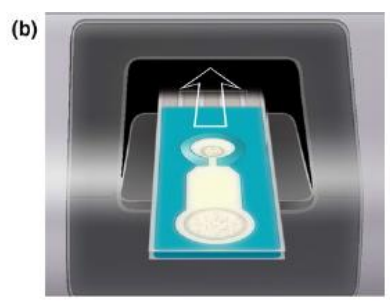

(c)

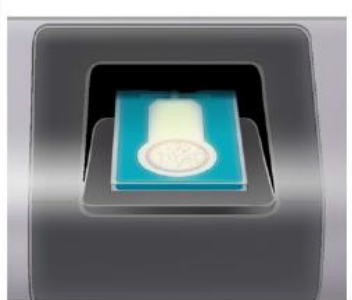

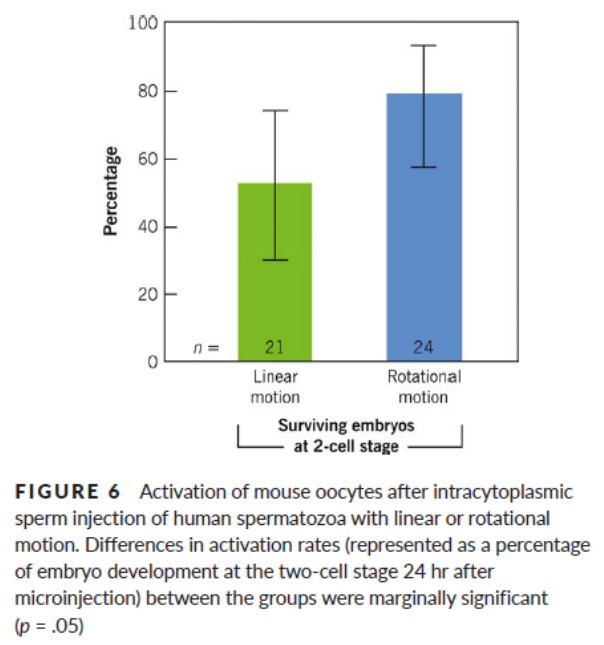

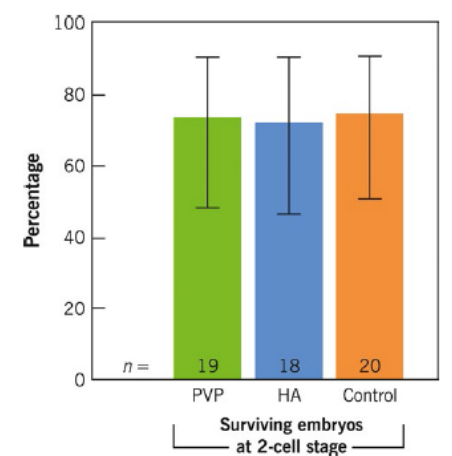

FIGURE 7 Activation of mouse oocytes after intracytoplasmic sperm injection of human spermatozoa selected in polyvinylpyrrolidone microdroplet, hyaluronic acid microdroplet or in culture medium (control). Differences in oocyte activation rates (represented as a percentage of embryo development at the two-cell stage $24 \mathrm{hr}$ after microinjection) were not significant among the groups

Our findings indicated a higher oocyte activation using spermatozoa with rotational motion than linear motion with an absolute difference of $27 \%$, which seems clinically important. However, given the small sample size and only marginally significant differences further studies are needed both in animal and human models to validate our results and to determine whether they translate in an improvement in other clinical outcomes.

We found that oocyte activations rates (recorded as the percentage of embryos at the two-cell stage $24 \mathrm{hr}$ after microinjection) were unaffected by the type of media, namely, PVP or HA, used for sperm handling before microinjection.

Nevertheless, the literature is mixed with regard to the use of PVP- and HA-based medium for sperm handling prior to ICSI (Balaban et al., 2003; Choe et al., 2012; Ebner, Filicori, Tews, \& Parmegiani, 2012; Majumdar \& Majumdar, 2013; Parmegiani, Cognigni, Bernardi, et al., 2010). In one study, Choe and colleagues suggested that ICSI outcomes are not related to the type of media used for sperm selection (Choe et al., 2012). The aforementioned authors enrolled 219 couples in which oocytes were injected with sperm selected either in PVP $(n=112)$ or HA $(n=107)$, and no significant differences were observed between the two groups in terms of fertilisation, cleavage and blastocyst formation. 


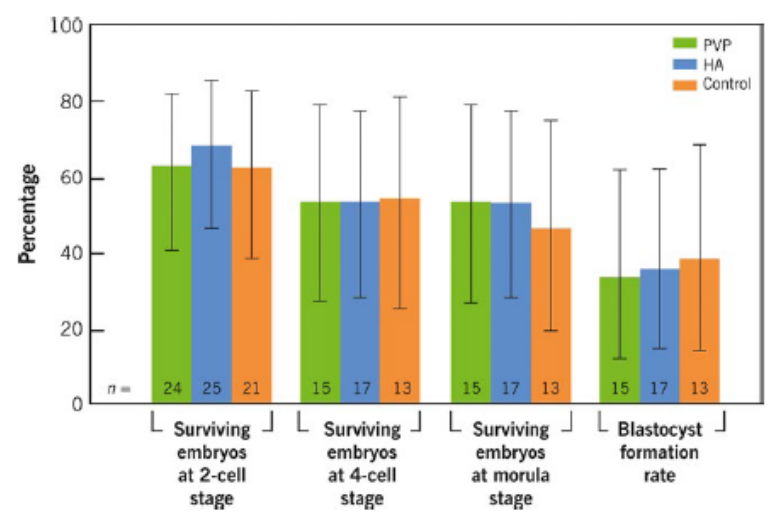

FIGURE 8 Embryo developmental competence of parthenogenetically activated mouse oocytes after intracytoplasmic sperm injection of polyvinylpyrrolidone, hyaluronic acid or culture medium (control). Differences in embryo development at the two-cell stage, four-cell stage, compact morula stage and blastocyst formation rates were not significant among the groups

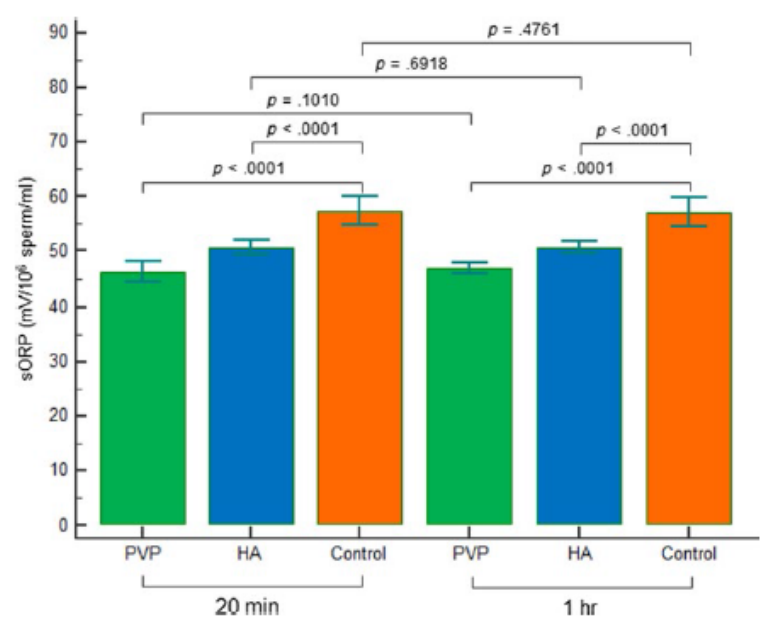

FIGURE 9 Oxidation-reduction potential of human spermatozoa selected for intracytoplasmic sperm injection after exposure to of polyvinylpyrrolidone, hyaluronic acid or culture medium (control) for $20 \mathrm{~min}$ and $1 \mathrm{hr}$. Differences in sORP were significantly different among the groups both at $20 \mathrm{~min}$ and $1 \mathrm{hr}(p<.05)$

However, cleavage rates on day 3 were significantly lower $(p=.038)$ in the HA group than the PVP group (Choe et al., 2012). In another study, Balaban et al. (2003) compared the outcomes of ICSI in 92 couples using PVP- $(n=44)$ and HA-selected $(n=48)$ spermatozoa and reported similar rates of fertilisation, cleavage, good quality embryos, clinical pregnancy and implantation. Despite that, the authors advocated the use of HA as an effective and more physiologic alternative to PVP to modulate sperm motility prior to aspirating a single spermatozoon into an ICSI pipette. Barak and coworkers also found similar rates of fertilisation, clinical pregnancy, implantation and live birth in 123 couples subjected to ICSI with spermatozoa selected in PVP $(n=65)$ or HA $(n=58)$ (Barak et al., 2001). Despite not finding any significant difference in ICSI outcomes, these authors commented that HA was a viable substitute to PVP based on the fact that it is a natural and readily degradable substance. These aforementioned results have been corroborated by recent studies (Majumdar \& Majumdar, 2013; Parmegiani, Cognigni, Bernardi, et al., 2010). On the contrary, NasrEsfahani et al. reported higher fertilisation rates $(79.4 \%$ vs. $67.7 \% ; p=.02)$ in the HA group 
compared to the PVP group, albeit the number of embryos generated, the percentages of cleavage and good quality embryo were not significantly different between the two groups on days 2 and 3 post-ICSI. Added to this, implantation rates and clinical pregnancy rates were not significantly different in this study (Nasr-Esfahani, Deemeh, \& Tavalaee, 2010). In a randomised control study, PVP and HA selection of spermatozoa was compared in 206 infertile couples treated by ICSI (Parmegiani, Cognigni, Bernardi, et al., 2010). The authors found that HA favoured sperm selection with lower levels of DNA fragmentation, as indicated by the sperm chromatin dispersion test, which might explain the higher proportion of embryos classified as having high quality (35.8\% vs. $24.1 \% ; p=.04)$ as well as the overall embryo development rate (95\% vs. $84 \% ; p \leq .001)$ in HAtreated specimens. However, these differences did not translate in any improvement in other clinical outcomes, including fertilisation rates, clinical pregnancy, implantation and live birth rates. The same group retrospectively analysed data from 86 infertile couples treated with PVP-ICSI and 293 couples with HA-ICSI in the same year and concluded that the proportion of embryos classified as having high quality ( $35.2 \%$ vs. $22.3 \% ; p=.01)$ and implantation rates (17.1\% vs. $10.3 \% ; p=.04)$ were higher in the HA group than the PVP group, even though no significant differences were observed in fertilisation rates, clinical pregnancy and live birth rates (Parmegiani, Cognigni, Ciampaglia, et al., 2010).

During ICSI, spermatozoa treated with the PVP solution can be easily immobilised by a light touch of the tail with a glass micropipette (Tsai et al., 2000). However, motility is resumed once spermatozoa are transferred to a normal culture medium (Dozortsev et al., 1995) if their tails are not completely broken, which might compromise oocyte activation. This indirect negative effect of PVP on sperm immobilisation has been suggested to be responsible for the decreased fertilisation rates shown in some studies rather than an arguable toxicity of PVP (Dozortsev et al., 1995).On the one hand, it has been proposed that sperm selection using HA is advantageous to prevent fertilisation of oocytes with DNA-damaged and chromosomal-unbalanced spermatozoa, which seems to be particularly relevant when specimens containing high proportion of defective spermatozoa are available for ICSI (Ebner et al., 2012). But the same might not be true when specimens taken from men with normal sperm parameters were used, as shown in our study. Furthermore, the ability of HA to deselect spermatozoa with damaged DNA is not unequivocal as recently shown (Huang et al., 2015).

The mouse oocyte activation test is a useful heterologous ICSI model to assess theimpact of PVP and HA on oocyte activation while controlling for the sperm factor (Heindryckx, De Gheselle, Gerris, Dhont, \& De Sutter, 2008; Heindryckx, Van Der Elst, De Sutter, \& Dhont, 2005; Rybouchkin et al., 1996; Tesarik, Rienzi, Ubaldi, Mendoza, \& Greco, 2002a,b). Calcium signalling is crucial for oocyte activation and further embryonic development (Ducibella, Schultz, \& Ozil, 2006; Kline \& Kline, 1992; Marangos \& Carroll, 2004; Ozil, Banrezes, Toth, Pan, \& Schultz, 2006). An increase in fertilisation and cleavage-stage embryo rates with the use of assisted oocyte activation protocols involving the use of $\mathrm{Ca}^{2+}$-ionophores has also been reported (Ebner et al., 2012; Heindryckx et al., 2008; Nikiforaki et al., 2016; Sfontouris et al., 2015; Vanden Meerschaut, Nikiforaki, Heindryckx, \& De Sutter, 2014).

This aforementioned model seems ideal to investigate whether PVP is toxic to oocytes and embryos. This is important because a small amount of PVP is unavoidably injected into the 
oocyte during sperm injections. Moreover, it has been suggested that PVP delays calcium oscillation in sperm-penetrated oocytes and prevents nuclear decondensation (Clarke \& Johnson, 1988; Dozortsev et al., 1995). Added to this, PVP is suggested to be associated with impaired two-cell mouse embryos development (Bergers et al., 1993) by exerting toxicity to mouse zygotes (Bras, 1994). While comparing ICSI outcomes after sperm selection in PVP or culture medium, Tsai et al. (2000) reported improved fertilisation rate (84.43\% vs. $57.63 \%$; $p<.001)$ and embryo grading $\left(\chi^{2}=6.80 ; p=.009\right)$ in the group where spermatozoa had been selected in the culture medium. Despite suggesting that elimination of PVP might be responsible for the observed improved fertilisation rate, these authors cautioned that other factors could be involved, including sperm factors. On the contrary, it has also been suggested that the biochemical and molecular parameters of developmental maturity displayed by spermatozoa selected in HA instead of PVP play a critical role in the paternal contribution to successful pre-implantation embryogenesis (Worrilow et al., 2013).

In our study, the metaphase II mouse oocytes were injected with PVP, HA or culture medium similar to a normal ICSI procedure. Oocytes were then activated parthenogenetically by exposure to a ready-to-use calcimycin $\left(\mathrm{Ca}^{2+}\right.$-selective ionophore) A23187 solution. Parthenogenetic oocyte activation and embryo development competence were recorded until blastocyst stage, and no significant differences among the PVP-treated, HA-treated and the control groupswere observed in oocyte degeneration rate, two- and four-cell embryo formation rate, compacted morula stage and blastocyst formation rate. To our knowledge, our results are novel in demonstrating that PVP and HA have similar effects on parthenogenetically activated mouse oocytes, adding confidence to the continued use of PVP in routine ICSI practice. The validation of our results using human oocytes is nevertheless required.

Seminal plasma is removed during semen processing for IVF and ICSI, and therefore, toxic oxygen metabolites (generated by immature sperm and residual leucocytes) may attack spermatozoa lacking the protection of seminal plasma antioxidants. As a matter of fact, the detrimental effect of oxidative stress on sperm functional competence can be further exaggerated by the in vitro sperm processing techniques, including density gradient centrifugation and prolonged incubation (Aitken \& Baker, 1995; De Iuliis et al., 2009; Zini et al., 2009), which might lead to sperm dysfunction and DNA damage (Aitken, De Iuliis, Finnie, Hedges, \& Mclachlan, 2010; Chen et al., 2012; Tremellen, 2008). The ORP is a comprehensive measure of oxidative stress as it provides the overall balance between all available oxidants and antioxidants in a given sample. Static ORP measures the current state of activity between oxidants and antioxidants, and it has been recently suggested that sORP of human spermatozoa could be potentially useful to predict ART outcomes, to monitor antioxidant levels and to identify infertile men who may benefit from antioxidant therapy as well as treatment monitoring (Agarwal, Roychoudhury, et al., 2016).

In our study, levels of sORP in human spermatozoa selected for ICSI were lower in the PVP-treated group than in the HA-treated group and the control group. These findings 
are also novel and suggest that PVP might alleviate sperm oxidative stress as it contains a synthetic serum replacement that acts as a chelating agent (Bavister, 1995; Nielsen \& Bertheussen,1991). This could be advantageous in the context of male factor infertility, as spermatozoa of infertile men usually have elevated oxidative stress. Of note, exposure of spermatozoa to hyaluronic acid alone has not shown to induce acrosome reaction (Hong et al., 2009; Sabeur, Cherr, Yudin, \& Overstreet, 1998). This is important because the acrosome reaction increases the production of ROS and would introduce a bias in our experiments. As shown in our experiment, the sORP levels remained unchanged after both short and prolonged sperm exposure to HA, thus adding confidence to our results. Our findings using a mouse model support the continued use of PVP for sperm selection and manipulation in ICSI processes. Notwithstanding, confirmatory experiments involving spermatozoa from infertile men are needed. Also important would be the assessment of the embryo development and implantation potential of resulting embryos from sperm populations exposed to variable time in PVP and HA and with measurable sORP levels.

\section{5 | Conclusions}

This series of experiments using an experimental ICSI model with mouse oocytes provides novel findings that add to the understanding of the sperm handling and selection step that precedes the sperm injection. For the first time, we demonstrated the selection of human spermatozoa with rotational motion as a novel indicator of sperm quality and it might also have the potential to improve ICSI outcome. Second, similar oocyte activation rates were achieved when spermatozoa were selected in a PVP- or HA-based medium. Third, we have shown in an experiment controlling for sperm factors that neither PVP nor HA seems to adversely affect development competence of parthenogenetically activated mouse oocytes. Lastly, measurement of sORP on washed spermatozoa selected for ICSI after exposure to PVP and HA revealed that the lowest levels of oxidative stress are found in spermatozoa selected in the PVP-based medium, thus suggesting possible antioxidant properties of PVP. Importantly, the results of the parameters analysed support the continued clinical use of PVP for selection and manipulation in ICSI processes.

\section{Acknowledgements}

The study was supported by funds from the American Center for Reproductive Medicine. Dr. Shubhadeep Roychoudhury was supported by the American Center for Reproductive Medicine and by a fellowship from the Department of Biotechnology, Government of India. The authors are grateful to Jeff Hammel, senior biostatistician, for his contribution to data analysis. Authors are grateful for the support of graphic artists from the Center for Medical Arts and Photography.

\section{ORCID}

S. Roychoudhury http://orcid.org/oooo-0oo3-4174-1852
A. Agarwal
http://orcid.org/oooo-0003-0585-1026
S. C. Esteves
http://orcid.org/oooo-0002-1313-9680
R. Henkel
http://orcid.org/oooo-0003-1128-2982
R. Sharma
http://orcid.org/oooo-0002-9664-6978 


\section{References}

Agarwal, A., Ahmad, G., \& Sharma, R. (2015). Reference values of reactive oxygen species in seminal ejaculates using chemiluminescence assay. Journal of Assisted Reproduction and Genetics, 32, 1721-1729. https:// doi.org/10.1007/s10815-015-0584-1

Agarwal, A., Mulgund, A., Alshahrani, S., Assidi, M., Abuzenadah, A. M., Sharma, R., \& Sabanegh, E. (2014). Reactive oxygen species and sperm DNA damage in infertile men presenting with low level leukocyto-spermia. Reproductive Biology and Endocrinology, 12, 126. https://doi. org/10.1186/1477-7827-12-126

Agarwal, A., Roychoudhury, S., Bjugstad, K. B., \& Cho, C. L. (2016). Oxidationreduction potential of semen: What is its role in the treatment of male infertility? Therapeutic Advances in Urology, 8, 302-318. https://doi.org/10.1177/1756287216652779

Agarwal, A., Sharma, R., Roychoudhury, S., Du Plessis, S., \& Sabanegh, E. (2016). MiOXSYS: A novel method of measuring oxidation reduction potential in semen and seminal plasma. Fertility and Sterility, 106, 566- 573 . https://doi.org/10.1016/j.fertnstert.2016.05.013

Aitken, R. J., \& Baker, H. W. (1995). Seminal leukocytes: Passengers, terrorists or good samaritans? Human Reproduction, 10, 1736-1739. https:// doi.org/10.1093/oxfordjournals.humrep.a136165

Aitken, R. J., De Iuliis, G. N., Finnie, J. M., Hedges, A., \& Mclachlan, R. I. (2010). Analysis of the relationships between oxidative stress, DNA damage and sperm vitality in a patient population: Development of diagnostic criteria. Human Reproduction, 25, 2415-2426. https://doi. org/10.1093/humrep/deq214

Balaban, B., Lundin, K., Morrell, J. M., Tjellstrom, H., Urman, B., \& Holmes, P. V. (2003). An alternative to PVP for slowing sperm prior to ICSI. Human Reproduction, 18, 18871889. https://doi.org/10.1093/humrep/deg385 Barak, Y., Menezo, Y., Veiga, A., \& Elder, K. (2001). A physiological replacement for polyvinylpyrrolidone (PVP) in assisted reproductive technology. Human Fertility (Cambridge, England), 4, 99-103. https://doi.org/10.1080/1464727012000199371

Bavister, B. D. (1995). Culture of preimplantation embryos: Facts and artifacts. Human Reproduction Update, 1, 91-148. https://doi.org/10.1093/ humupd/1.2.91

Bergers, J., Dumoulin, J., Bras, M., Pieters, E., Geraedts, P., \& Evers, J. (1993). The effect of polymers on the cryopreservation of mouse embryos. Human Reproduction, 8, 148.

Bourne, H., Richings, N., Liu, D. Y., Clarke, G. N., Harari, O., \& Baker, H. W. (1995). Sperm preparation for intracytoplasmic injection: Methods and relationship to fertilization results. Reproduction, Fertility, and Development, 7, 177-183. https://doi.org/10.1071/RD9950177

Bras, M. (1994). The use of a mouse zygote quality control system for training purposes and toxicity determination in an ICSI programme. Human Reproduction, 9, 23-24.

Bungum, L., Bungum, M., Humaidan, P., \& Andersen, C. Y. (2004). A strategy for treatment of couples with unexplained infertility who failed to conceive after intrauterine insemination. Reproductive BioMedicine Online, 8, 584-589. https://doi.org/10.1016/ S1472-6483(10)61107-8 
Cayli, S., Jakab, A., Ovari, L., Delpiano, E., Celik-Ozenci, C., Sakkas, D., ... Huszar, G. (2003). Biochemical markers of sperm function: Male fertility and sperm selection for ICSI. Reproductive BioMedicine Online, 7, 462-468. https://doi.org/10.1016/S14726483(10)61891-3

Chen, H., Zhao, H. X., Huang, X. F., Chen, G. W., Yang, Z. X., Sun, W. J., ... Shi, H. J. (2012). Does high load of oxidants in human semen contribute to male factor infertility? $\begin{array}{lllll}\text { Antioxidants } \quad \text { and } & \text { Redox }\end{array}$ https://doi.org/10.1089/ars.2011.4461

Choe, S. A., Tae, J. C., Shin, M. Y., Kim, H. J., Kim, C. H., Lee, J. Y., ... Jee, B. C. (2012). Application of sperm selection using hyaluronic acid binding in intracytoplasmic sperm injection cycles: A sibling oocyte study. Journal of Korean Medical Science, 27, 1569-1573. https://doi.org/10.3346/ jkms.2012.27.12.1569

Clarke, R., \& Johnson, L. (1988). Factors related to successful sperm micro-injection of hamster eggs: The effect of sperm species, technical experience, needle dimensions, and incubation medium on egg viability and sperm decondensation following microinjection. Theriogenology, 30, 447-460. https://doi.org/10.1016/0093691X(88)90194-X

De Iuliis, G. N., Thomson, L. K., Mitchell, L. A., Finnie, J. M., Koppers, A. J., Hedges, A., ... Aitken, R. J. (2009). DNA damage in human spermatozoa is highly correlated with the efficiency of chromatin remodeling and the formation of 8-hydroxy-2'deoxyguanosine, a marker of oxidative stress. Biology of Reproduction, 81, 517-524. https://doi.org/10.1095/ biolreprod.109.076836

Devroey, P., \& Van Steirteghem, A. (2004). A review of ten years experience of ICSI. Human Reproduction Update, 10, 19-28. https://doi. org/10.1093/humupd/dmhoo4

Dozortsev, D., Rybouchkin, A., De Sutter, P., Qian, C., \& Dhont, M. (1995). Human oocyte activation following intracytoplasmic injection: The role of the sperm cell. Human Reproduction, 10, 403-407. https://doi. org/10.1093/oxfordjournals.humrep.a135952

Ducibella, T., Schultz, R. M., \& Ozil, J. P. (2006). Role of calcium signals in early development. Seminars in Cell and Developmental Biology, 17, 324-332. https://doi.org/10.1016/j.semcdb.2006.02.010

Ebner, T., Filicori, M., Tews, G., \& Parmegiani, L. (2012). A plea for a more physiological ICSI. Andrologia, 44(Suppl 1), 2-19. https://doi. org/10.1111/j.14390272.2011.01266.x

Flaherty, S. P., Payne, D., \& Matthews, C. D. (1998). Fertilization failures and abnormal fertilization after intracytoplasmic sperm injection. Human Reproduction, 13(Suppl 1), 155-164. https://doi.org/10.1093/ humrep/13.suppl_1.155

Heindryckx, B., De Gheselle, S., Gerris, J., Dhont, M., \& De Sutter, P. (2008). Efficiency of assisted oocyte activation as a solution for failed intracytoplasmic sperm injection. Reproductive BioMedicine Online, 17, 662- 668. https://doi.org/10.1016/S14726483(10)60313-6

Heindryckx, B., Van Der Elst, J., De Sutter, P., \& Dhont, M. (2005). Treatment option for spermor oocyte-related fertilization failure: Assisted oocyte activation following diagnostic heterologous ICSI. Human Reproduction, 2O, 2237-2241. https://doi.org/10.1093/humrep/deio29 
Hong, S. J., Chiu, P. C., Lee, K. F., Tse, J. Y., Ho, P. C., \& Yeung, W. S. (2009). Cumulus cells and their extracellular matrix affect the quality of the spermatozoa penetrating the cumulus mass. Fertility and Sterility, 92, 971-978. https://doi.org/10.1016/j.fertnstert.2008.07.1760

Huang, M. T., Kuo-Kuang Lee, R., Lu, C. H., Chen, Y. J., Li, S. H., \& Hwu, Y. M. (2015). The efficiency of conventional microscopic selectionis comparable to the hyaluronic acid binding method in selecting spermatozoa for male infertility patients. Taiwanese Journal of Obstetrics and Gynecology, 54, 48-53. https://doi.org/10.1016/j. tjog.2014.11.006

Huszar, G., Jakab, A., Sakkas, D., Ozenci, C. C., Cayli, S., Delpiano, E., \& Ozkavukcu, S. (2007). Fertility testing and ICSI sperm selection by hyaluronic acid binding: Clinical and genetic aspects. Reproductive BioMedicine Online, 14, 650-663. https://doi.org/10.1016/ S1472-6483(10)61060-7

Kashir, J., Heindryckx, B., Jones, C., De Sutter, P., Parrington, J., \& Coward, K. (2010). Oocyte activation, phospholipase $\mathrm{C}$ zeta and human infertility. Human Reproduction Update, 16, 690-703. https://doi.org/10.1093/ humupd/dmq018

Kato, Y., \& Nagao, Y. (2012). Effect of polyvinylpyrrolidone on sperm function and early embryonic development following intracytoplasmic sperm injection in human assisted reproduction. Reproductive Medicine and Biology, 11, 165-176. https://doi.org/10.1007/ s12522-012-0126-9

Kimura, Y., Yanagimachi, R., Kuretake, S., Bortkiewicz, H., Perry, A. C., \& Yanagimachi, H. (1998). Analysis of mouse oocyte activation suggests the involvement of sperm perinuclear material. Biology of Reproduction, 58, 1407-1415. https://doi.org/10.1095/biolreprod58.6.1407

Kline, D., \& Kline, J. T. (1992). Repetitive calcium transients and the role of calcium in exocytosis and cell cycle activation in the mouse egg. Developmental Biology, 149, 80-89. https://doi. org/10.1016/0012-1606(92)90265-I

Majumdar, G., \& Majumdar, A. (2013). A prospective randomized study to evaluate the effect of hyaluronic acid sperm selection on the in-tracytoplasmic sperm injection outcome of patients with unexplained infertility having normal semen parameters. Journal of Assisted Reproduction and Genetics, 30, 1471-1475. https://doi.org/10.1007/ s10815-013-0108-9

Marangos, P., \& Carroll, J. (2004). Fertilization and InsP3-induced Ca2+ release stimulate a persistent increase in the rate of degradation of cyclin B1 specifically in mature mouse oocytes. Developmental Biology, 272, 26-38. https://doi.org/10.1016/j.ydbio.2004.04.012

Mason, M. J., \& Grinstein, S. (1993). Ionomycin activates electrogenic Ca2+ influx in rat thymic lymphocytes. Biochemical Journal, 296(Pt 1), 33-39. https://doi.org/10.1042/bj2960033

Miki, K., \& Clapham, D. E. (2013). Rheotaxis guides mammalian sperm. Current Biology, 23, 443-452. https://doi.org/10.1016/j. cub.2013.02.007

Montag, M., Toth, B., \& Strowitzki, T. (2012). Sperm selection in ART. Journal of Reproductive Medicine and Endocrinology, 9, 485-489. 
Morgan, A. J., \& Jacob, R. (1994). Ionomycin enhances Ca2+ influx by stimulating storeregulated cation entry and not by a direct action at the plasma membrane. Biochemical Journal, 30o(Pt 3), 665-672. https:// doi.org/10.1042/bj3000665

Motoishi, M., Goto, K., Tomita, K., Ookutsu, S., \& Nakanishi, Y. (1996). Examination of the safety of intracytoplasmic injection procedures by using bovine zygotes. Human Reproduction,11, 618-620.https://doi. org/10.1093/HUMREP/11.3.618

Nasr-Esfahani, M. H., Deemeh, M. R., \& Tavalaee, M. (2010). Artificial oocyte activation and intracytoplasmic sperm injection. Fertility and Sterility, 94, 520-526. https://doi.org/10.1016/j.fertnstert.2009.03.061

Neri, Q. V., Lee, B., Rosenwaks, Z., Machaca, K., \& Palermo, G. D. (2014). Understanding fertilization through intracytoplasmic sperm injection (ICSI). Cell Calcium, 55, 2437. https://doi.org/10.1016/j. ceca.2013.10.006

Nielsen, H. I., \& Bertheussen, K. (1991). A protein free serum supplement (SSR) for cell culture. In R. E. Spier, J. B. Griffiths \& B. Meignier (Eds.), Production of biologicals from animal cells in culture (pp. 187-189). Oxford, UK: Butterworth-Heinemann Ltd, Halley Court.

Nikiforaki, D., Vanden Meerschaut, F., de Roo, C., Lu, Y., Ferrer-Buitrago, M., de Sutter, P., \& Heindryckx, B. (2016). Effect of two assisted oocyte activation protocols used to overcome fertilization failure on the activation potential and calcium releasing pattern. Fertility and Sterility, 105, 798-806. https://doi.org/10.1016/j.fertnstert.2015.11.007

Nosrati, R., Driouchi, A., Yip, C. M., \& Sinton, D. (2015). Two-dimensional slither swimming of sperm within a micrometre of a surface. Nature Communications, 6, 8703. https://doi.org/10.1038/ncomms9703

Ozil, J. P., Banrezes, B., Toth, S., Pan, H., \& Schultz, R. M. (2006). $\mathrm{Ca}^{2+}$ oscillatory pattern in fertilized mouse eggs affects gene expression and development to term. Developmental Biology, 300, 534-544. https:// doi.org/10.1016/j.ydbio.2006.08.041

Ozil, J. P., Markoulaki, S., Toth, S., Matson, S., Banrezes, B., Knott, J. G., ... Ducibella, T. (2005). Egg activation events are regulated by the duration of a sustained $\left[\mathrm{Ca}^{2+}\right]$ cyt signal in the mouse. Developmental Biology, 282, 39-54. https://doi.org/10.1016/j.ydbio.2005.02.035

Parmegiani, L., Cognigni, G. E., Bernardi, S., Troilo, E., Ciampaglia, W., \& Filicori, M. (2010). "Physiologic ICSI": Hyaluronic acid (HA) favors selection of spermatozoa without DNA fragmentation and with normal nucleus, resulting in improvement of embryo quality. Fertility and Sterility, 93, 598-604. https://doi.org/10.1016/j.fertnstert.2009.03.033

Parmegiani, L., Cognigni, G. E., Ciampaglia, W., Pocognoli, P., Marchi, F., \& Filicori, M. (2010). Efficiency of hyaluronic acid (HA) sperm selection. Journal of Assisted Reproduction and Genetics, 27, 13-16. https://doi. org/10.1007/s10815-009-9380-0

Ray, B. D., Howell, R. T., Mcdermott, A., \& Hull, M. G. (1995). Testing the mutagenic potential of polyvinylpyrrolidone and methyl cellulose by sister chromatid exchange analysis prior to use in intracytoplasmic sperm injection procedures. Human Reproduction, 10, 436-438. https://doi.org/10.1093/oxfordjournals.humrep.a135957 
Roychoudhury, S., Sharma, R., Sikka, S., \& Agarwal, A. (2016). Diagnostic application of total antioxidant capacity in seminal plasma to assess oxidative stress in male factor infertility. Journal of Assisted Reproduction and Genetics, 33, 627-635. https://doi.org/10.1007/s10815-016-0677-5

Rybouchkin, A., Dozortsev, D., Pelinck, M. J., De Sutter, P., \& Dhont, M. (1996). Analysis of the oocyte activating capacity and chromosomal complement of round-headed human spermatozoa by their injection into mouse oocytes. Human Reproduction, 11, 2170-2175. https://doi. org/10.1093/oxfordjournals.humrep.a019071

Sabeur, K., Cherr, G. N., Yudin, A. I., \& Overstreet, J. W. (1998). Hyaluronic Acid enhances induction of the acrosome reaction of human sperm through interaction with the $\mathrm{PH}-20 \quad$ protein. Zygote, 2, $103-111$. https://doi.org/10.1017/So967199498000021

Sfontouris, I. A., Nastri, C. O., Lima, M. L., Tahmasbpourmarzouni, E., Raine-Fenning, N., \& Martins, W. P. (2015). Artificial oocyte activation to improve reproductive outcomes in women with previous fertilization failure: Asystematic review and meta-analysis of RCTs. Human Reproduction, 30, 1831-1841. https://doi.org/10.1093/humrep/dev136

Subramani, E., Basu, H., Thangaraju, S., \& Dandekar, S. (2014). Rotational dynamics of optically trapped human spermatozoa. Scientific World Journal, 2014, 154367. https://doi.org/10.1155/2014/154367

Swain, J. E., \& Pool, T. B. (2008). ART failure: Oocyte contributions to unsuccessful fertilization. Human Reproduction Update, 14, 431-446. https://doi.org/10.1093/humupd/dmno25

Takeuchi, S., Minoura, H., Shibahara, T., Shen, X., Futamura, N., \& Toyoda, N. (2000). In vitro fertilization and intracytoplasmic sperm injection for couples with unexplained infertility after failed direct intraperitoneal insemination. Journal of Assisted Reproduction and Genetics, 17, 515- 520. https://doi.org/10.1023/A:1009445909023

Tesarik, J., Rienzi, L., Ubaldi, F., Mendoza, C., \& Greco, E. (2002). Use of a modified intracytoplasmic sperm injection technique to overcome sperm-borne and oocyteborne oocyte activation failures. Fertility and Sterility, 78, 619-624. https://doi.org/10.1016/ So015-0282(02)03291-0

Tesarik, J., Sousa, M., \& Testart, J. (1994). Human oocyte activation after Intracytoplasmic sperm injection. Human Reproduction, 9, 511-518. https://doi.org/10.1093/oxfordjournals.humrep.a138537

Toth, S., Huneau, D., Banrezes, B., \& Ozil, J. P. (2006). Egg activation is the result of calcium signal summation in the mouse. Reproduction, 131, 27-34. https://doi.org/10.1530/rep.1.00764

Tremellen, K. (2008). Oxidative stress and male infertility-a clinical perspective. Human Reproduction Update, 14, 243-258. https://doi. org/10.1093/humupd/dmno04

Tsai, M. Y., Huang, F. J., Kung, F. T., Lin, Y. C., Chang, S. Y., Wu, J. F., \& Chang, H. W. (2000). Influence of polyvinylpyrrolidone on the outcome of intracytoplasmic sperm injection. Journal of Reproductive Medicine, 45, 115-120.

Uranga, J. A., Pedersen, R. A., \& Arechaga, J. (1996). Parthenogenetic activation of mouse oocytes using calcium ionophores and protein kinase $\mathrm{C}$ stimulators. International Journal of Developmental Biology, 40, 515-519. 
Vanden Meerschaut, F., Nikiforaki, D., Heindryckx, B., \& De Sutter, P. (2014). Assisted oocyte activation following ICSI fertilization failure. Reproductive BioMedicine Online, 28, 560-571. https://doi. org/10.1016/j.rbmo.2014.01.008

Worrilow, K. C., Eid, S., Woodhouse, D., Perloe, M., Smith, S., Witmyer, J., ... Lieberman, J. (2013). Use of hyaluronan in the selection of sperm for intracytoplasmic sperm injection (ICSI): Significant improvement in clinical outcomes-multicenter, double-blinded and randomized controlled trial. Human Reproduction, 28, 306-314. https://doi.org/10.1093/humrep/ des417

Yanagida, K. (2004). Complete fertilization failure in ICSI. Human Cell, 17, 187-193.

Zini, A., San Gabriel, M., \& Baazeem, A. (2009). Antioxidants and sperm DNA damage: A clinical perspective. Journal of Assisted Reproduction and Genetics, 26, 427-432. https://doi.org/10.1007/s10815-009-9343-5 